\title{
Penetration and exchange kinetics of primary alkyl amines applied to reactive poly(pentafluorophenyl acrylate) thin films
}

\author{
Hyunjoo Son ${ }^{1,4}$, Yeongseon Jang ${ }^{1,4,5}$, Jaseung Koo $^{2}$, Jeong-Soo Lee ${ }^{2}$, Patrick Theato ${ }^{3}$ and Kookheon Char ${ }^{1}$
}

We investigated the mechanism of primary amine-induced post-polymerization modification of active ester polymer thin films composed of pentafluorophenyl acrylate (PFPA) polymers. The most important physical parameters in the post-polymerization modification are the molecular weight of the PFPA polymers and the aliphatic chain length of the primary amines. The effects of these two parameters on the penetration depth, as well as the exchange kinetics were systematically studied by neutron reflectivity and quartz crystal microbalance with dissipation, accompanied by the measurement of surface morphological changes by an atomic-force microscopy and optical microscopy. The spin-cast PFPA polymer thin films showed distinctive differences in the exchange kinetics, as well as the penetration depth of amines as a function of the PFPA polymer molecular weight. The aliphatic chain length of primary alkyl amines markedly influenced the penetration kinetics into low-molecular weight poly(PFPA) films, whereas there was no significant penetration effect on the high-molecular weight films. The high-molecular weight of the poly(PFPA) films led to the deceleration of the dissolution of amine-functionalized polymer chains in a good solvent. The results of this study may provide a good physical background for developing reactive poly(PFPA) thin film platforms based on simple and quantitative post-modification with amine-containing molecules.

Polymer Journal (2016) 48, 487-495; doi:10.1038/pj.2016.6; published online 10 February 2016

\section{INTRODUCTION}

Reactive polymer thin films have recently received substantial attention for many potential applications such as bio-functional membranes ${ }^{1,2}$ and stimuli-responsive coatings with controlled wettability. ${ }^{3,4}$ Polymers carrying activated ester groups are known to be good candidates owing to their high reactivity with primary or secondary amines. One active ester polymer, poly(pentafluorophenyl acrylate) (poly(PFPA)), has been highlighted as a reactive polymer that facilitates the post-polymerization modification. Facile postmodification of pentafluorophenyl esters via amine-containing functional groups allows for convenient and versatile functionalization of diverse polymer platforms including brushes ${ }^{5,6}$ and thin films. ${ }^{7-10}$

In particular, the functionalized thin films based on this active esteramine chemistry have offered an easy approach to realizing diverse functions by simple reactions with quantitative conversion. For example, the post-polymerization modification of poly(PFPA) films with amines has been utilized to obtain free-standing robust fluorescent films by layer-by-layer assembly based on covalent bonds between active ester polymers and poly(allyl amine), ${ }^{10}$ as well as functionalized thin films composed of photocleavable block copolymers with a vertically oriented nanoporous structure. ${ }^{9}$ Complete conversion through the entire film thickness was initially inferred by analyzing the components of the films with photoluminescence, NMR or FT-IR spectra. However, very little has been investigated thus far about the exchange mechanism and kinetics of primary alkyl amines applied to poly(PFPA) thin films. To obtain more insights on the post-modification of thin films, an in-depth understanding of the internal structural changes of polymer films triggered by active ester-amine chemistry was required for practical applications.

Herein, we report the detailed internal structural changes in the amine-treated poly(PFPA) thin films as a function of the aliphatic chain length of the primary amines, as well as the molecular weight of the poly(PFPA). The poly(PFPA) thin films investigated in this study were prepared in the form of spin-cast thin films. Because the spin-coating method has the ability to quickly and easily produce well-defined films, ${ }^{11,12}$ the spin-cast thin films of poly(PFPA) could be used as model systems. In addition, the interactions between solvent and polymer films have primarily been studied to gain insights regarding polymer film systems in the case of solution processes,

\footnotetext{
${ }^{1}$ The National Creative Research Initiative Center for Intelligent Hybrids, The WCU Program of Chemical Convergence for Energy and Environment, School of Chemical and Biological Engineering, Seoul National University, Seoul, Korea; ${ }^{2}$ Neutron Science Division, Korea Atomic Energy Research Institute, Daejeon, Korea and ${ }^{3}$ Department of Chemistry, Institute for Technical and Macromolecular Chemistry, University of Hamburg, Hamburg, Germany

4These authors contributed equally to this work.

${ }^{5}$ Current address: School of Chemical and Biomolecular Engineering, Geargia Institute of Technology, Atlanta, 30332 GA, USA.

Correspondence: Professor K Char, The National Creative Research Initiative Center for Intelligent Hybrids, The WCU Program of Chemical Convergence for Energy and Environment, School of Chemical and Biological Engineering, Seoul National University, Gwanak 1, Gwanak-ro, Gwanak-Gu, Seoul 08826, Korea.

E-mail: khchar@plaza.snu.ac.kr

Received 11 November 2015; revised 21 December 2015; accepted 21 December 2015; published online 10 February 2016
} 
such as the penetration of solvent molecules and the dissolution behavior of polymer thin films. ${ }^{13-15}$ Similarly, it is important to monitor the status of functionalized poly(PFPA) films because postpolymerization modification should be performed in an aminecontaining solution.

Thin films of high- and low-molecular weight poly(PFPA) synthesized by reversible addition-fragmentation chain transfer (RAFT) polymerization were treated post-polymerization with simple primary alkyl amines of different alkyl chain lengths. We conducted quartz crystal microbalance with dissipation (QCM-D) monitoring to determine the amine-exchange kinetics in situ and in real-time, combined with atomic-force microscopy and optical microscopy to observe the changes in the surface morphology. Furthermore, the changes in the film thickness and internal structure of poly(PFPA) films after aminetreatment were observed with neutron reflectivity (NR) measurements, which are regarded as a powerful tool to study the material interfaces in nanometer-scale thin films. ${ }^{12}$

\section{EXPERIMENTAL PROCEDURE}

\section{Materials}

All chemicals and solvents were purchased from Sigma-Aldrich and used as received, except for acryloyl chloride, which was purchased from the Tokyo Chemical Industry (Tokyo, Japan). Benzyl dithiobenzoate, used as the RAFT chain transfer agent, was synthesized according to a previously published method. ${ }^{16}$ Silica gel for column chromatography was purchased from Merck Chemical Company (Darmstadt, Germany). Silicon wafers (100) and Au sensor crystals (QSX301, Q-Sense) were used as substrates to prepare the polymer thin films.

\section{Synthesis of PFPA}

Pentafluorophenol (15.3 g, $83.1 \mathrm{~mm})$ and $10.0 \mathrm{~g}(98.8 \mathrm{~mm})$ of triethylamine were dissolved in $150 \mathrm{ml}$ of diethyl ether, and $8.95 \mathrm{~g}(98.9 \mathrm{~mm})$ of acryloyl chloride was added dropwise through a funnel under cooling with an ice bath. The solution was stirred for $1 \mathrm{~h}$ in an ice bath and then stirred overnight at room temperature. After the precipitated salt was separated by filtration, the solvent was evaporated and the crude product was purified by column chromatography (column material: silica gel; solvent: petroleum ether). A colorless liquid $(15.8 \mathrm{~g}, 66.4 \mathrm{~mm}, 80 \%)$ was obtained. ${ }^{1} \mathrm{H}$ NMR $\left(\mathrm{CDCl}_{3}\right)$ : S/p.p.m.: 6.74 (d, 1H), 6.39 (dd, 1H), 6.19 (d, 1H). ${ }^{19} \mathrm{~F}$ NMR $\left(\mathrm{CDCl}_{3}\right):-161$ (d, 2 F), $-156.70(\mathrm{t}, 1 \mathrm{~F}),-151.40$ (d, 2 F). FT-IR: $1772 \mathrm{~cm}^{-1}(\mathrm{C}=\mathrm{O}$ ester band), $1516 \mathrm{~cm}^{-1}$ ( $\mathrm{C}=\mathrm{C}$ aromatic band). The detailed procedure has been well-documented in previous studies. ${ }^{17,18}$

\section{Synthesis of Poly(PFPA) via RAFT polymerization}

A $1.13 \mathrm{~g}(4.72 \mathrm{~mm})$ aliquot of PFPA, $5.0 \mathrm{mg}(0.0205 \mathrm{~mm})$ of benzyl dithiobenzoate, $0.4 \mathrm{mg}(0.00244 \mathrm{~mm})$ of 2,2'-azobis(2-methylpropionitrile) and $3.0 \mathrm{ml}$ of anisole were placed into a Schlenk flask. After three freeze-pumpthaw cycles, the flask filled with nitrogen gas was stirred in an oil bath at $70{ }^{\circ} \mathrm{C}$ for $4 \mathrm{~h}$ and then cooled down to room temperature. The polymer was precipitated into methanol twice and dried in a vacuum oven. ${ }^{1} \mathrm{H}$ NMR $\left(\mathrm{CDCl}_{3}\right)$ : $\delta$ /p.p.m.: 3.10 (br, s), 2.5 (br, s), 2.13 (br, s). ${ }^{19} \mathrm{~F}$ NMR $\left(\mathrm{CDCl}_{3}\right)$ : -164 (br, s), -158.6 (br, s), -155 (br, s).

\section{Poly(PFPA) thin film preparation and post-polymerization treatment with primary alkyl amines}

Silicon wafers (100) were cleaned using piranha solution $\left(70 \% \mathrm{H}_{2} \mathrm{SO}_{4}\right.$ and $30 \%$ $\mathrm{H}_{2} \mathrm{O}_{2}$ ) for $20 \mathrm{~min}$, rinsed with deionized water and dried with a nitrogen stream. Poly(PFPA) thin films were deposited by the spin-coating method using $3 \mathrm{wt} \%$ of poly(PFPA) solution in tetrahydrofuran with a spin-rate of 3000 r.p.m. for 30 s.

High- $\left(\mathrm{M}_{\mathrm{w}}=170 \mathrm{~kg} \mathrm{~mol}^{-1}\right)$ and low-molecular weight $\left(\mathrm{M}_{\mathrm{w}}=37 \mathrm{~kg} \mathrm{~mol}^{-1}\right)$ poly(PFPA) thin films were prepared, and post-polymerization modification was performed by dipping the films in a $1 \mathrm{wt} \%$ solution of primary alkyl amine. Each alkyl amine with a different alkyl chain length (amylamine and dodecylamine) was dissolved in ethanol. The post-polymerization treated films were washed thoroughly with ethanol, and all films were dried under a stream of nitrogen.

\section{Characterization}

${ }^{1} \mathrm{H}$ NMR spectra and ${ }^{19} \mathrm{~F}$ NMR spectra were recorded on a Bruker Avance $500 \mathrm{MHz}$ FT-NMR spectrometer (Bruker, Billerica, MA, USA). Chemical shifts were given in p.p.m. relative to trimethylsilane. Gel permeation chromatography was used to determine the molecular weight and the corresponding polydispersity index $\left(\mathrm{PDI}=\mathrm{M}_{\mathrm{w}} / \mathrm{M}_{\mathrm{n}}\right)$ of the polymer samples. Gel permeation chromatography (YL9100, Young Lin Instrument, Anyang, Korea) measurements were performed under poly(styrene) standards in tetrahydrofuran with a $5 \mathrm{mg} \mathrm{ml}^{-1}$ polymer sample concentration.

The film thickness was obtained by a variable-angle multiwavelength ellipsometer (Gaertner L2W15S830, Gaertner Scientific, Skokie, IL, USA). The film surface morphology was monitored by tapping-mode atomic-force microscopy (Innova AFM, Veeco, Plainview, NY, USA) combined with an optical microscope. Every film treated with amines for a specific reaction time $(0,20,40$ and $60 \mathrm{~min})$ was characterized under air after thorough washing with ethanol followed by gentle drying with a nitrogen stream.

The QCM-D measurements (Q-Sense D300, Q-Sense) were performed to monitor the changes in frequency $\left(\Delta \mathrm{f}_{n}\right)$ and dissipation energy $\left(\Delta D_{n}\right)$ of an $A u$ sensor crystal (QSX301) coated with poly(PFPA) during the chemical reaction. The changes in frequency $\left(\Delta \mathrm{f}_{\mathrm{n}}\right)$ are proportional to the changes in mass, according to the Sauerbrey equation. ${ }^{19}$ The dissipation energy shift $\left(\Delta D_{n}\right)$ indicates the loss of energy stored in the vibration cycle, which yields useful information on the changes in the viscoelastic properties as well as the structural transformation. ${ }^{11,12}$ As-prepared poly(PFPA) films were immersed in a poor solvent, ethanol, for $1 \mathrm{~h}$ in order to set the baseline to monitor the amine-treated changes in the QCM frequency and dissipation energy in the solvent setting. After the stabilization, $0.5 \mathrm{ml}$ of amine solution was injected into the sample chamber. The applied voltage was sequentially pulsed across the $\mathrm{Au}$ sensor crystal, allowing the shear wave to dissipate as well as the simultaneous measurement of the absolute dissipation and absolute resonant frequency of the crystal for all four overtones $(n=1,3,5$ and 7, that is, 5, 15, 25 and $35 \mathrm{MHz}$ ). All of the measurements were taken at $25^{\circ} \mathrm{C}$. Because $\Delta \mathrm{f}_{1}$ and $\Delta \mathrm{D}_{1}$ were typically noisy owing to insufficient energy trapping, the frequency changes in the third overtone $\left(\Delta \mathrm{f}_{3} / 3\right)$ were compared between the samples.

The internal structures of post-treated poly(PFPA) films were characterized by NR. Samples for NR experiments were prepared on 3-inch diameter and 5 -mm thick silicon wafers. All measurements were performed at room temperature. NR measurements were conducted with a vertical reflectometer at the High-flux Advanced Neutron Application Reactor (HANARO) of the Korea Atomic Energy Research Institute in Daejeon, Korea. The neutron wavelength $(\lambda)$ was $4.75 \AA$, with $\Delta \lambda / \lambda$ equal to $\sim 0.02$. Scattering from samples was corrected for background, and the reflectivity curves were fitted to obtain the depth profile by using Parratt 32 and Motofit reflectivity analysis packages. The resulting scattering length density (SLD) profiles defined the zero of film thickness as the interface between a polymer and a native oxide layer. The film thickness was calculated based on the model layers of polymer films.

\section{RESULTS AND DISCUSSION}

\section{Post-polymerization modification of poly(PFPA) thin films with} primary alkyl amines

Poly(PFPA) was synthesized by RAFT polymerization with a desired molecular weight $\left(\mathrm{M}_{\mathrm{W}}=37 \mathrm{~kg} \mathrm{~mol}^{-1}\right)$ and a relatively narrow polydispersity index $(\mathrm{PDI}=1.3)$. An additional functionalization process of the poly(PFPA) thin films was triggered by an aminolysis reaction with primary alkyl amines dissolved in ethanol, which is classified as a poor solvent for poly(PFPA). The aminolysis reaction of the poly(PFPA) with primary alkyl amines is presented in Figure 1a. In order to understand the post-polymerization modification process of poly(PFPA) films $\left(\mathrm{M}_{\mathrm{W}}=37 \mathrm{~kg} \mathrm{~mol}^{-1}\right)$ with a dodecylamine $\left(\mathrm{CH}_{3}\right.$ $\left.\left(\mathrm{CH}_{2}\right)_{11} \mathrm{NH}_{2}\right)$ solution, we monitored the changes in film thickness as well as surface morphology as a function of the post-polymerization 
a<smiles>CC(C)(C)CC(C(=O)Oc1c(F)c(F)c(F)c(F)c1F)C(C)(C)C(=O)O</smiles><smiles>CC(C)C(C(=O)O)C(=O)N1CCCCCCCC1</smiles>

Poly $(N$-alkylacrylamide $)$

\section{b}
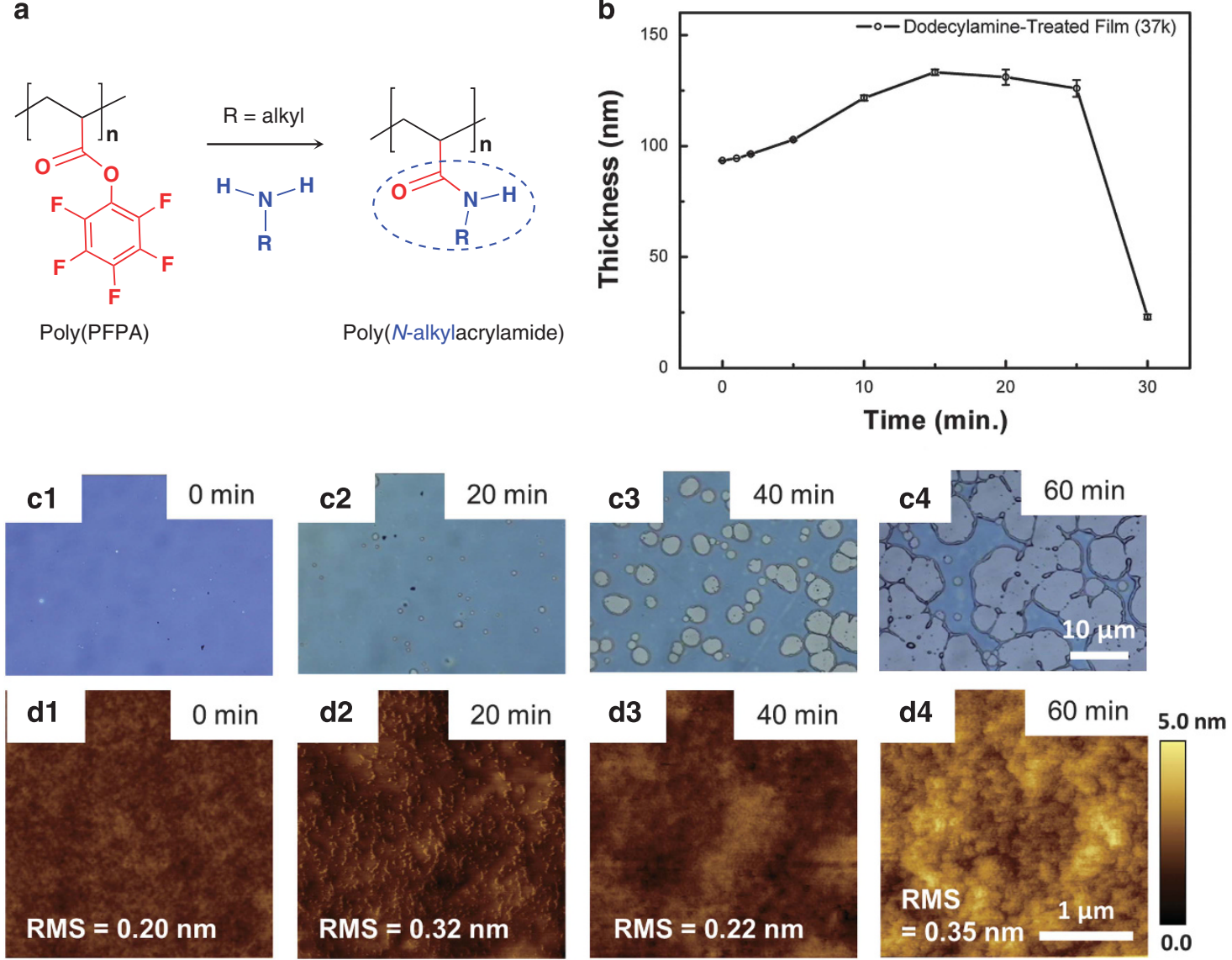

Figure 1 (a) Chemical structures of the reactive poly(PFPA) and primary alkyl amine used in this study. The aminolysis reaction results in the product (poly ( $N$-alkyl acrylamide). (b) Thickness changes in poly(PFPA) films $\left(M_{W}=37 \mathrm{~kg} \mathrm{~mol}^{-1}\right)$ treated with dodecylamine post-polymerization. The total film thickness was measured by ellipsometry under air before the dewetting began. The error bars indicate the standard deviation of three independent measurements. (c) Optical microscope images and (d) tapping-mode AFM height images of poly(PFPA) films ( $\mathrm{M}_{\mathrm{W}}=37 \mathrm{~kg} \mathrm{~mol}^{-1}$ ) after 0 (c1, d1), 20 (c2, d2), 40 (c3, d3) and $60 \mathrm{~min}(\mathbf{c 4}, \mathrm{d} 4)$ treatments with dodecylamines. AFM images were obtained from areas where no dewetting was observed.

treatment time. Figure $1 \mathrm{~b}$ shows that the thickness of postpolymerization treated films slowly increased initially, followed by a rapid decrease in the film thickness. The initial spin-cast poly(PFPA) films had relatively smooth ( $\mathrm{rms}$ roughness $=0.20 \mathrm{~nm}$ ) and flat surfaces before the post-polymerization treatment. However, the film surfaces gradually developed irregular patterns of droplets as the dipping time of the films in the amine solution was increased (Figures 1c,d).

Polymer dissolution in good solvents is characterized by the following common stages in sequence: the penetration of solvent molecules into polymer films, leading to the relaxation of the polymers; the formation of a solvent-swollen gel layer; and the diffusion of polymer chains into the solvent. ${ }^{15}$ Although ethanol is a poor solvent for poly(PFPA), the films showed ordinary dissolution behavior in a good solvent during the post-polymerization treatment with dodecylamines. This indicates that the amine molecules used as substituents change the solubility of polymer chains during the postpolymerization modification in an ethanol solution. We hypothesize that the amine molecules penetrated into the excluded volume of poly (PFPA) films and formed a swollen amine-substituted polymer gel layer, which finally led to the dewetting behavior followed by dissolution in ethanol. In addition, the substitution of pentafluorophenyl groups in poly(PFPA) with amine moieties occurred directly after the penetration of amines and solvent molecules owing to the high reactivity of poly(PFPA) with primary amines. However, further studies are needed to clearly elucidate the detailed mechanism of the penetration and substitution of amines in poly(PFPA) films as well as the swelling and dissolution of the resulting poly $(N$-alkyl acrylamide) films in the solution state. In this regard, we studied the postpolymerization modification kinetics of the poly(PFPA) films as a function of the aliphatic chain length of the primary alkyl amine.

Effect of the aliphatic chain length of the primary alkyl amines on the exchange kinetics of poly(PFPA) films

We monitored the changes in surface morphology during the postpolymerization modification with amylamine containing five carbons $\left(\mathrm{CH}_{3}\left(\mathrm{CH}_{2}\right)_{4} \mathrm{NH}_{2}\right)$ in order to examine the effect of aliphatic chain length on the substitution kinetics for reactive groups in the poly (PFPA) thin films $\left(\mathrm{M}_{\mathrm{W}}=37 \mathrm{~kg} \mathrm{~mol}^{-1}\right)$ and compared the results to treatment with dodecylamine.

The poly(PFPA) films treated in an amylamine solution for $10 \mathrm{~min}$ revealed the bare silicon wafer owing to the diffusion of the resulting amine-substituted polymers in ethanol. However, dodecylaminetreated poly(PFPA) films remained on the silicon wafers after the same post-polymerization treatment time $(10 \mathrm{~min})$, whereas showing small holes in the swollen state, as confirmed by the increase in film thickness, as well as changes in the surface morphology (Figures 2a and $\mathrm{b}$ ). This indicated that the shorter primary alkyl amines induced 
a

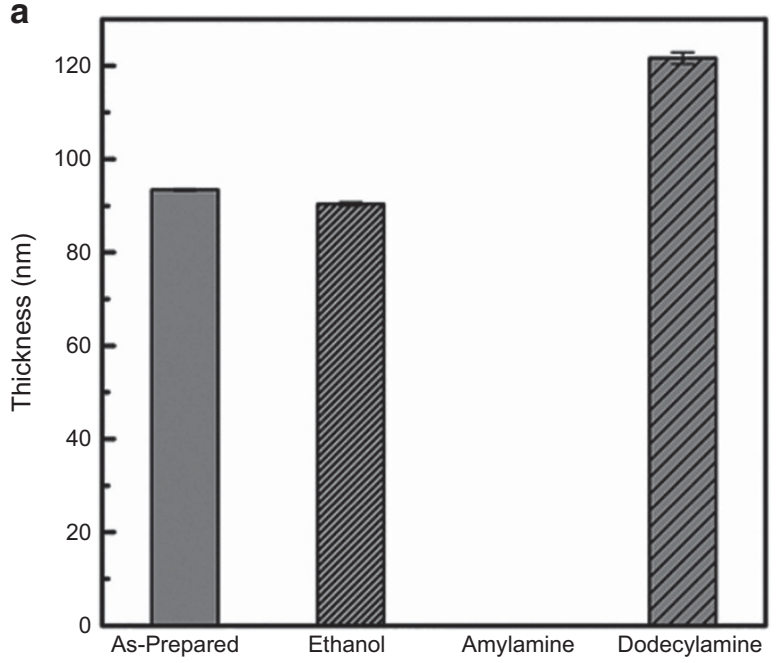

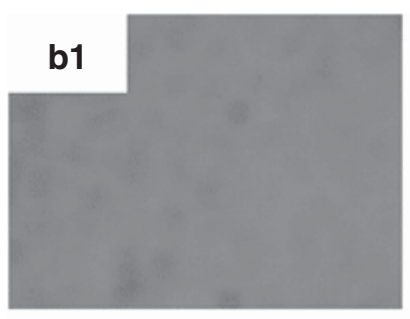
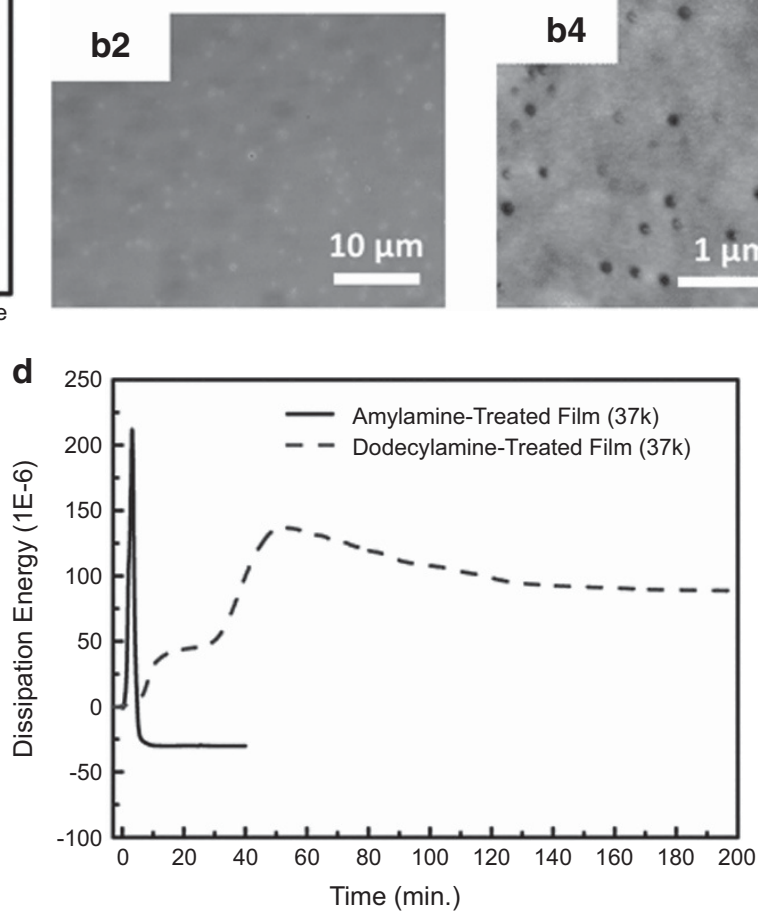

Figure 2 (a) Film thicknesses of poly(PFPA) films $\left(M_{w}=37 \mathrm{~kg} \mathrm{~mol}^{-1}\right)$ as-prepared and the films immersed in ethanol, amylamine-ethanol solution and dodecylamine-ethanol solution for $10 \mathrm{~min}$. (b) Optical microscope and AFM images of the poly(PFPA) films after 10 min treatment with amylamine (b1, b3) and dodecylamine (b2, b4). QCM-D monitoring of the changes in (c) frequency and (d) dissipation energy of the poly(PFPA) films $\left(\mathrm{M}_{\mathrm{w}}=37 \mathrm{~kg} \mathrm{~mol}^{-1}\right.$ ) post-treated with amylamine (solid line) and dodecylamine (dashed line). A full color version of this figure is available at Polymer Journal online.

much faster exchanges with pentafluorophenyl side groups in the polymers leading to faster dissolution of the poly(PFPA) films when compared with longer primary alkyl amines. This result suggests that the exchange kinetics of poly(PFPA) films can be fine-tuned by using primary alkyl amines with different aliphatic chain lengths.

To elucidate the detailed mechanism of the swelling and dissolution kinetics of poly(PFPA) films treated with two different types of amines, we performed QCM-D monitoring, as shown in Figures $2 \mathrm{c}$,d. Amylamine quickly reacted with the pentafluorophenyl groups of poly(PFPA) films as soon as the amylamines dissolved in ethanol were injected into a QCM-D cell containing a poly(PFPA)coated substrate. Initially, a negative frequency shift of the amylaminetreated film indicated a small gain in mass, which originated from the binding of amylamines to the polymer in a short time (Figure 2c). After the rapid substitution of the amines into poly(PFPA) films, they rapidly formed poly( $N$-pentyl acrylamide) that was readily soluble in ethanol, thus resulting in successive loss in mass, as confirmed by a significant increase in the frequency shift after $10 \mathrm{~min}$ of treatment.

In addition, the initial rapid increase in the dissipation energy demonstrated the formation of swollen layers on the top film surface (Figure $2 \mathrm{~d}$ ). The amylamine molecules quickly penetrated into the poly(PFPA) films, and the interactions between the reacted polymer chains and ethanol became more favorable. We note that the kinetics from the QCM-D results support the rapid penetration and spontaneous substitution of amylamines into the modified film, triggering the quick dissolution of the film, as confirmed by the microscopic image of the exposed bare silicon wafer after $10 \mathrm{~min}$ of treatment.

However, in the case of the post-polymerization treatment with dodecylamines with 12 carbons in the alkyl chain, the QCM-D curves demonstrated much slower penetration and dissolution kinetics owing to the longer retention time of a swollen polymer gel layer. Dodecylamines slowly penetrated into the films for $20 \mathrm{~min}$ owing to their larger size, whereas amylamine soaked into the films quickly. The mass of dodecylamine-treated polymer films gradually increased during the initial $15 \mathrm{~min}$ of treatment. We believe that this is because the excluded volume of the polymer layer was gradually increased as dodecylamines and solvent molecules slowly penetrated into the films. Moreover, the dodecylamine-treated film represents a two-step increase in dissipation energy. This indicates that dodecylamines could not readily penetrate into the film but were adsorbed on the surface upon reaction initially, developing soft top layers interacting with the ethanol solvent. After the slow propagation of the swollen layer into the entire film by the full sorption of dodecylamines for $40 \mathrm{~min}$, the dodecylamine-modified polymer layers started to dissolve in ethanol. 
In addition, the QCM-D results exactly corresponded to the film thickness and surface morphology results: an initial increase in film thickness at $10 \mathrm{~min}$, owing to the adsorption of dodecylamines, the growth of holes on the film surface at $40 \mathrm{~min}$, and the resulting dewetting at $60 \mathrm{~min}$, as shown in Figures $1 \mathrm{~b}, \mathrm{c}$.

From the observed changes in the poly(PFPA) film mass, softness, film thickness and surface morphology as a function of the treatment time with different amines, we traced the detailed post-modification mechanism of reactive poly(PFPA) films with primary alkyl amines. In addition, we clearly demonstrated that the kinetics in this sequential reaction can be regulated by the aliphatic chain length of the substituting primary amine. The longer primary amines led to slower penetration and a slower substitution rate in well-defined spin-cast poly(PFPA) films compared with their shorter amine counterparts. The dewetting and dissolution kinetics also slowed as the aliphatic chain length of primary amines increased. Because ethanol is a poor solvent for poly(PFPA), the spin-cast poly(PFPA) films had very welldefined and tightly collapsed surfaces when in contact with pure ethanol. However, once amine molecules reached or contacted the film, amines penetrated into the films and underwent a substitution reaction altering the solubility of the film in ethanol. In the penetration and substitution steps, the size of primary amines critically determined the formation rate and the solubility of the substituted poly( $\mathrm{N}$-alkyl acrylamide). Furthermore, the molecular weight of the primary amines used for the post-polymerization modification determined not only the size of the substituents but also their molar concentration. The molar concentration of amylamines was approximately two times higher than that of dodecylamines for the same amine solution mass concentration (that is, $1 \mathrm{wt} \%$ in this study). Therefore, amine molecules with lower molecular weights can accelerate the penetration into and exchange kinetics with poly(PFPA) films owing to both their smaller size and higher molar concentration.

Nevertheless, the ultimate dissolution of polymer thin films after the post-polymerization treatment makes it hard to utilize and functionalize poly(PFPA) thin films by using the easy substitution with functional primary alkyl amines. Furthermore, more information on the control of the amine penetration depth and degree of substitution is required to realize functional surfaces. Hence, in order to identify physical parameters to tune the degree of substitution of amines into poly(PFPA) thin films as well as to prevent the dissolution of aminemodified films, we further investigated the effect of the molecular weight of poly(PFPA) on the dissolution.

\section{Effect of the molecular weight of poly(PFPA) chains on the dissolution of PPFPA thin films by post-treatment with primary amines}

The molecular weight of the polymer film is another important physical parameter controlling the degree of functionalization of poly(PFPA) films. Two different poly(PFPA) films with low $\left(\mathrm{M}_{\mathrm{W}}=37 \mathrm{~kg} \mathrm{~mol}^{-1}\right)$ and high- $\left(\mathrm{M}_{\mathrm{W}}=170 \mathrm{~kg} \mathrm{~mol}^{-1}\right)$ molecular weights were synthesized to investigate the molecular weight effect on the post-polymerization modification with simple aliphatic amines. Along with the kinetics obtained from QCM-D measurements, NR was additionally used to gain information on the internal structural changes of films after amine-triggered post-polymerization treatment. $\mathrm{NR}$ is the most advantageous tool to investigate the buried structures and interfacial roughness on a nanometer-scale within a polymer thin film, which contains strong neutron-scattering contrast layers. Because the SLD of poly(PFPA) is much higher than that of aminemodified poly( $N$-alkyl acrylamide) owing to the neutron-rich pentafluorophenyl side groups $\left(\mathrm{SLD}_{\text {poly }(\mathrm{PFPA})}=3.5-3.8 \times 10^{-4} \mathrm{~nm}^{-2}\right.$,
$\left.\operatorname{SLD}_{\text {poly }(N \text {-alkyl acrylamide })}=0.3-0.6 \times 10^{-4} \mathrm{~nm}^{-2}\right)$, we were able to obtain enough information on the penetration depth of amines into the film, as well as the resulting swollen polymer gel layer thickness of poly $(N$-alkyl acrylamide) without additional deuteration of polymers for NR measurements.

We demonstrated that poly(PFPA) films of lower molecular weight $\left(\mathrm{M}_{\mathrm{w}}=37 \mathrm{~kg} \mathrm{~mol}^{-1}\right)$ showed distinctly different kinetics in post-polymerization modification reactions according to the aliphatic chain length of the primary amines. NR measurements were conducted to analyze the component changes in the polymer films before and after the post-polymerization treatment as a function of the molecular weight of the poly(PFPA) and aliphatic chain length of the primary amines. In order to verify the detailed post-modification mechanism suggested by QCM-D, microscopy and thickness monitoring, the internal structures of the films were systematically investigated by $\mathrm{NR}$ at specific post-treatment times $(0,3,5$ and $10 \mathrm{~min})$, which were selected from the swelling time region identified in the QCM-D experiments.

As shown in Figure 3a, the SLD values of amylamine-treated films significantly decreased as the treatment time increased, demonstrating that amylamine molecules vigorously penetrated into the lowmolecular weight films $\left(\mathrm{M}_{\mathrm{w}}=37 \mathrm{~kg} \mathrm{~mol}^{-1}\right)$. After $5 \mathrm{~min}$ of treatment with amylamines, the post-polymerization treated film showed an $\sim 24.8 \%$ reduction in the total thickness $(73.3 \mathrm{~nm})$ when compared with the original film thickness $(97.5 \mathrm{~nm})$. The amylamine molecules penetrated into $40.8 \%$ of the initial film thickness after $5 \mathrm{~min}$ of treatment, which was estimated from the ratio of the thickness of the remaining poly(PFPA) layer $(57.7 \mathrm{~nm})$ with respect to the original film thickness. Because the penetration of amine molecules triggers the decrease in the SLD value from the polymer-air interface, the layer of unreacted poly(PFPA) was defined as the layer that had the SLD value of poly(PFPA) $\left(3.5-3.8 \times 10^{-4} \mathrm{~nm}^{-2}\right)$, calculated from the fitted model layers. The approximate degree of functionalization of poly (PFPA) films was estimated from the SLD reduction (\%), the percentage ratio of the area under the SLD curves of as-prepared and post-polymerization treated polymer films. The SLD reduction originates from the substitution of pentafluorophenyl groups with $N$-pentyl groups that have a much lower SLD value. From this point of view, a $25.5 \%$ reduction in the SLD of the 5 min-treated film implies quantitative changes in the internal components. In addition, the low-molecular weight polymers with reactive side groups that were exchanged with amylamines were rapidly dissolved in ethanol from the top film surface within $\sim 10 \mathrm{~min}$. After $10 \mathrm{~min}$ of treatment, only a 5.3-nm thickness of the polymer film was left with a $0.3-\mathrm{nm}$ thickness of unreacted poly(PFPA), resulting in a SLD reduction of up to $94.6 \%$. The changes in film structure and internal components of the amylamine-treated low-molecular weight film occurred so rapidly owing to the fast penetration and exchange kinetics of short amylamines as well as the rapid dissolution of the resulting low-molecular weight poly( $N$-pentyl acrylamide).

The results with higher molecular weight $\left(M_{W}=170 \mathrm{~kg} \mathrm{~mol}^{-1}\right)$ poly (PFPA) films prompted us to investigate the interpenetration depth profile of amylamines into the poly(PFPA) films with NR, which allowed us to understand exactly what happens inside the film (Figure 3b). The amylamine-treated high-molecular weight polymer films maintained the total film thickness while demonstrating the amine penetration depth and the interlayer roughness as a function of post-polymerization treatment time in the fitted SLD profiles. High-molecular weight poly(PFPA) chains were almost intact at the top film surface even after the exchange with alkyl amines, which was confirmed by the fact that there was virtually no significant change in 
a1

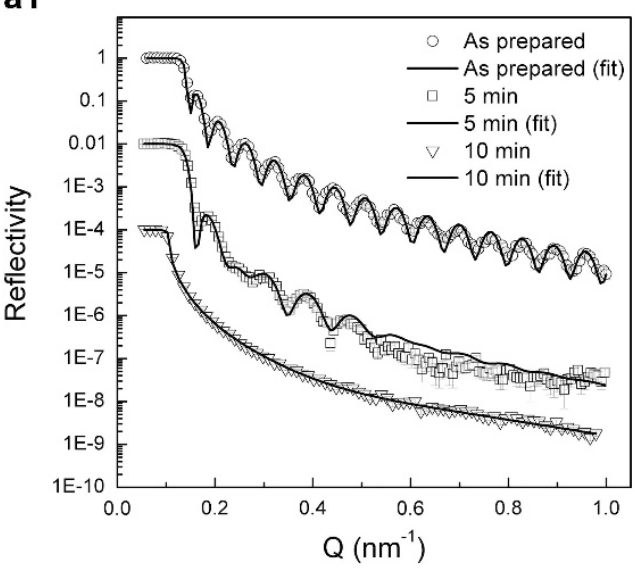

b1

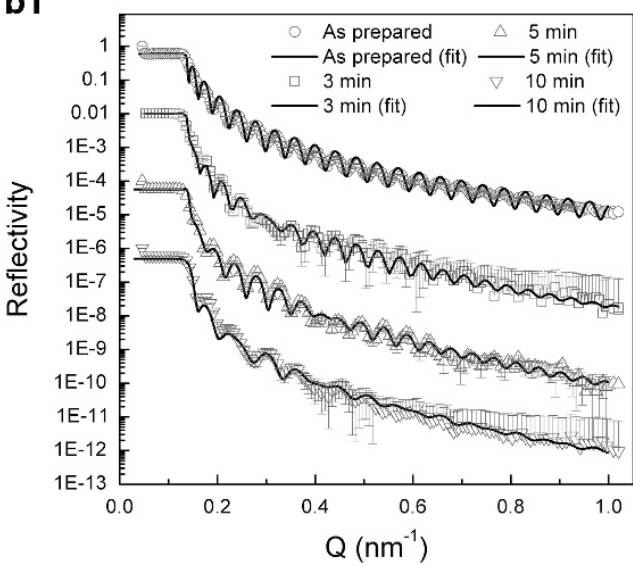

a2

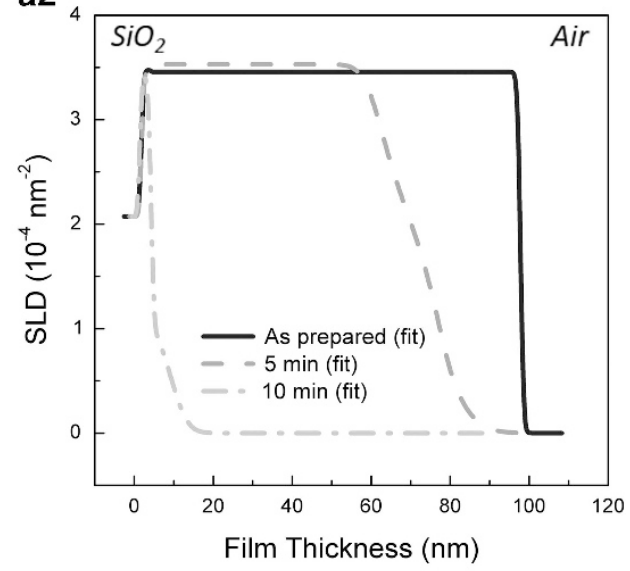

b2

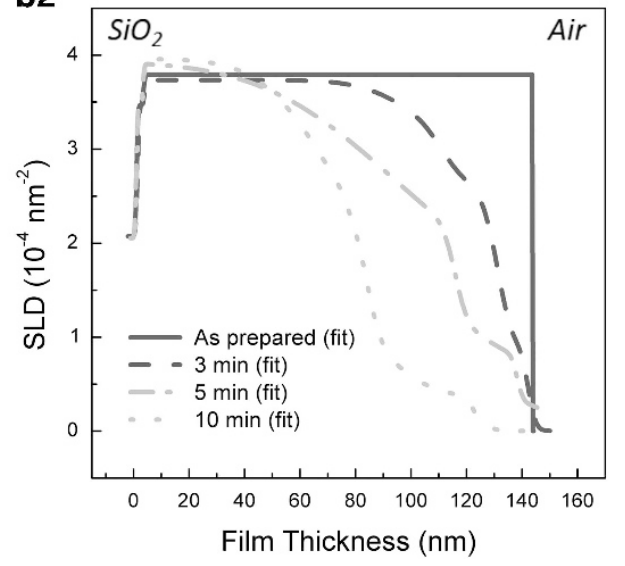

Figure 3 Neutron reflectivity curves and SLD profiles of the as-prepared and post-polymerization treated poly(PFPA) films of (a) low-molecular weight $\left(37 \mathrm{~kg} \mathrm{~mol}^{-1}\right)$ and (b) high-molecular weight $\left(170 \mathrm{~kg} \mathrm{~mol}^{-1}\right)$ after amylamine-treatment for different treatment times.

the film thickness of the poly(PFPA) film in the SLD curve after the post-polymerization treatment for $10 \mathrm{~min}$. However, amylamine molecules gradually penetrated into the film. As a result, the exchanged product poly( $N$-pentyl acrylamide) was formed as a swollen gel layer, but this dissolved much more slowly in ethanol owing to the increased entanglements between the longer polymer chains. Along the same lines, 5 min-treated poly(PFPA) films had an amine-functionalized layer $45.8-\mathrm{nm}$ thick, which meant that $36.5 \%$ of the initial film thickness was penetrated by amylamine, accompanied with a $25.4 \%$ SLD reduction. For the additional 5 min of treatment, the SLD reduction increased up to $42.3 \%$, which was accompanied by a $50.8 \%$ amine penetration depth into the film, whereas the dry-film thickness of poly(PFPA) films changed slightly from $134.8 \mathrm{~nm}$ (5 min) to $120.5 \mathrm{~nm}$ after $10 \mathrm{~min}$ of treatment (that is, 3.7 and $13.9 \%$ decreased with respect to the original film thickness $(140.0 \mathrm{~nm})$ ).

When we compared the data from 5-min treatment with amylamine between the two different molecular weight polymer films, the $40.8 \%$ amylamine penetration depth into the low-molecular weight polymer film was similar to the $36.5 \%$ penetration depth in the highmolecular weight film. However, the film thickness of the lowmolecular weight film was significantly decreased (24.7\%) compared with a slight drop (3.7\%) in the film thickness in the case of the highmolecular weight film because the dissolution behavior of the lowmolecular weight polymer film was accelerated by the instant swelling of the exchanged layer. However, the high-molecular weight polymer films maintained the entire thin film structure even after postpolymerization treatment with amylamines. These results indicated that short primary amines can readily penetrate into polymer films regardless of the molecular weight of the polymers, whereas the dissolution of amine-modified polymer films is wholly dependent on the polymer molecular weight.

In contrast to the amylamine modification, the post-polymerization treatment with dodecylamines showed different behavior of penetration and exchange in poly(PFPA) films, as well as a different dependency on the polymer molecular weight. As shown in Figures 4a and b, QCM-D data demonstrated that the highmolecular weight polymer film showed an increase in mass and the development of a soft layer at the top surface owing to amine binding and the formation of a swelling layer, whereas the low-molecular weight film was finally dissolved in ethanol after amine penetration. Moreover, the dodecylamine-treated low-molecular weight film had an equilibrium in the swelling region from 20 to $40 \mathrm{~min}$ before disintegration, whereas the high-molecular weight film did not dissolve even after swelling with amine exchange.

The fact that dodecylamine has a lower diffusion rate than amylamine makes the difference in the SLD profiles as a function of post-polymerization treatment time noteworthy. Compared with the penetration profiles of amylamine, dodecylamine molecules soaked into the poly(PFPA) film at a much slower rate and dodecylamines that could not rapidly penetrate in a short time were simply bound to 

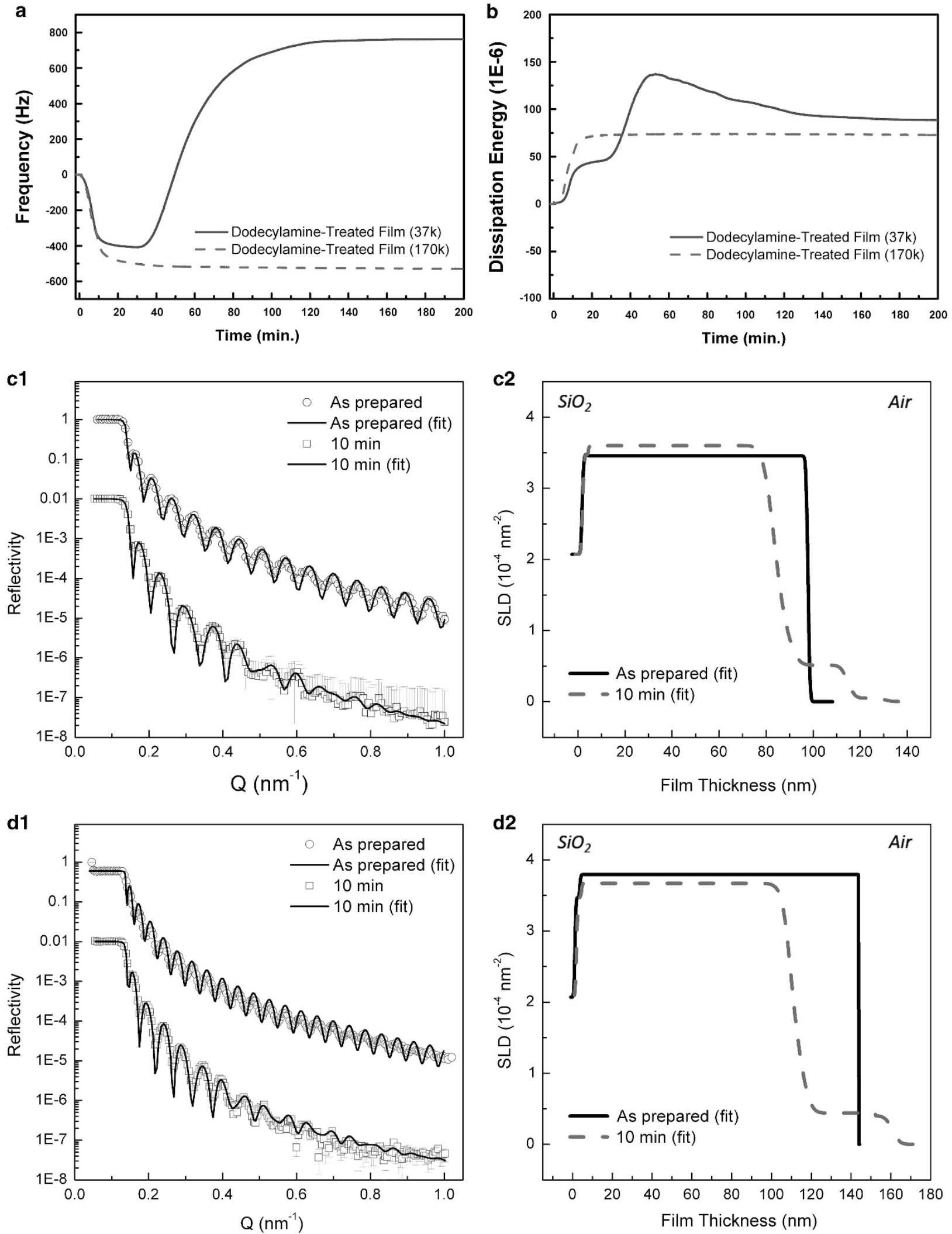

Figure 4 QCM-D data showing the changes in (a) frequency and (b) dissipation energy of the poly(PFPA) films with low (37 $\mathrm{kg} \mathrm{mol}^{-1}$; solid line) and high (170 $\mathrm{kg} \mathrm{mol}^{-1}$; dashed line) molecular weights after treated with dodecylamines. Neutron reflectivity curves and SLD profiles of the as-prepared and post-polymerization treated poly(PFPA) films of (c) low-molecular weight $\left(37 \mathrm{~kg} \mathrm{~mol}^{-1}\right)$ (d) high-molecular weight $\left(170 \mathrm{~kg} \mathrm{~mol}^{-1}\right)$ after treatment with dodecylamines. 
the surface regardless of the polymer molecular weight. The swollen layer of dodecylamine-treated polymers yielded an increased film thickness in both molecular weight cases because of its longer alkyl chain moieties. Nonetheless, the final dissolution behavior of the product in ethanol was determined by the molecular weight.

In more detail, low-molecular weight $\left(\mathrm{M}_{\mathrm{W}}=37 \mathrm{~kg} \mathrm{~mol}^{-1}\right)$ poly (PFPA) thin films exhibited a retardation in the penetration rate of dodecylamines, but the exchange process finally led to the complete dewetting of the films. Figure $4 \mathrm{c}$ indicates the formation of a swollen layer after $10 \mathrm{~min}$ of treatment and a $29.4 \%$ increased film thickness with respect to the original film thickness $(97.5 \mathrm{~nm})$. Fitted model layers of the NR curves showed that dodecylamine penetrated $19.2 \%$ of the initial film thickness, as estimated from the thickness of unreacted poly(PFPA) $(78.8 \mathrm{~nm})$, and a $5.4 \%$ reduction of the SLD value. However, the dewetting eventually occurred after the films were fully exchanged with amines and swollen, as observed from the QCM-D data. Poly(PFPA) films of low-molecular weight after longer treatment times with dodecylamine could not be measured with NR owing to the severe roughening of the surfaces of the polymer films.

However, the dissolution process was apparently limited in the high-molecular weight polymer films even after the formation of the top swollen layer consisting of poly( $N$-alkyl acrylamide). As shown in Figure $4 \mathrm{~d}$, the thickness of the post-polymerization treated film increased by $11.6 \%(156.2 \mathrm{~nm})$ compared with the thickness of the as-prepared film $(140.0 \mathrm{~nm})$. Despite the increase in the total film thickness, the SLD value was reduced up to $21.3 \%$ in the entire film, demonstrating that dodecylamine penetrated $37.1 \%$ of the initial film thickness. Even after the first 10 min of treatment, most of the amine-functionalized polymer chains remained in the polymer matrix with a continuous increase in the thickness of the swollen layer at the surface. For the same reaction time, dodecylamine molecules penetrated more deeply into the polymer film when the film was composed of a higher molecular weight poly(PFPA). The increase in the film thickness was induced by the reaction of dodecylamines with poly (PFPA) chains and the corresponding formation of longer alkyl side chains. The entanglements of high-molecular weight polymer chains within the thin film also prevent the dissolution of the polymers after the aminolysis reaction. ${ }^{20}$ In addition, it has been well-documented that the tightly packed surfaces of spin-cast films also have an important role in controlling the degree and rate of penetration and dissolution of the solvent as a function of the molecular weight. ${ }^{12}$ This indicates that amine-modified functional polymer thin films can simply be realized by the aminolysis reaction of high-molecular weight poly(PFPA) films by soaking functional amines dissolved in solvent. Moreover, the penetration depth of the amines and the corresponding degree of functionalization in high-molecular weight reactive thin films can be controlled by the aliphatic chain length of the primary amines.

On the basis of the observations of the surface morphologies on the nanometer- and micrometer-scale, the film mass and softness, and the internal layered structures, we identify the size of the primary alkyl amine as a new factor affecting the disentanglement of the initial polymer thin film and the solvent diffusion, which are important contributors to the dissolution of polymers. ${ }^{15}$ The post-modification process in the poly(PFPA) films with primary alkyl amines is conducted through three main steps: adsorption and penetration of amine and solvent molecules, the aminolysis reaction and the diffusion or dissolution of the resulting poly( $N$-alkyl acrylamide) chains in solution. The degree of amine penetration, as well as the modification rate can be tuned by the size of the alkyl length in the amine molecules, whereas the dissolution kinetics are mainly controlled by the molecular weight of the reactive polymers leading to the backbone structure in thin films. Consequently, the high-molecular weight reactive poly(PFPA) films have great potential for controlled post-polymerization modification via functional amines without the loss of films in a treatment solution.

\section{CONCLUSION}

The spin-cast poly(PFPA) films were subjected to post-polymerization modification based on their fast reactivity with primary amines. Either amylamines or dodecylamines, with different alkyl chain lengths, were used for the post-polymerization modification of the poly(PFPA) thin films. The results demonstrated that amine molecules penetrate into the films from the top surface, making the films swollen owing to the high-affinity between amine-modified polymers and the solvent (ethanol). The rate of amine penetration and exchange in poly(PFPA) films was primarily determined by the aliphatic chain length of the primary amines employed for the post-polymerization treatment. Primary amines with longer alkyl chain lengths led to slower penetration and exchange in the poly(PFPA) films. It was also observed that the short amylamine-modified polymer films with low-molecular weight were rapidly dissolved in the solvent. However, the dissolution behavior of amine-treated polymer films could be reduced with an increase in the molecular weight of the poly(PFPA) in thin films. As a consequence, we could easily realize the aminefunctionalized thin films by spin-casting high-molecular weight poly (PFPA) films and immersing them in an amine-containing ethanol solution for $10 \mathrm{~min}$ without causing the dissolution of the polymer films. This fundamental study demonstrating the effects of the aliphatic chain length in the primary amines, as well as the molecular weight of the reactive polymers would provide important physical parameters for the functionalization of polymer thin films in a solvent based on the chemical substitution of side groups. Furthermore, based on the fundamental information provided in this study, the reactive poly(PFPA) thin films may be further extended into diverse functional platforms through versatile post-modifications with a number of amines that have different functionalities.

\section{CONFLICT OF INTEREST}

The authors declare no conflict of interest.

\section{ACKNOWLEDGEMENTS}

This work was financially supported by the National Research Foundation of Korea (NRF) funded by the Korea Ministry of Science, ICT \& Future Planning (MSIP) (The National Creative Research Initiative Program for 'Intelligent Hybrids Research Center' (No. 2010-0018290), the BK21 Plus Program in SNU Chemical Engineering, and the WCU Program of Chemical Convergence for Energy and Environment (R31-10013)). We thank the HANARO Center of Korea Atomic Energy Research Institute (KAERI) for assigning enough beam time to conduct the NR experiments at the REF-V beam line. HS and YJ contributed equally to this work.

1 Ulbricht, M. Advanced functional polymer membranes. Polymer 47, 2217-2262 (2006).

2 Deng, X., Eyster, T. W., Elkasabi, Y. \& Lahann, J. Bio-orthogonal polymer coatings for co-presentation of biomolecules. Macromol. Rapid. Commun. 33, 640-645 (2012).

3 Kessler, D., Jochum, F. D., Choi, J., Char, K. \& Theato, P. Reactive surface coatings based on polysilsesquioxanes: universal method toward light-responsive surfaces. ACS Appl. Mater. Interfaces 3, 124-128 (2011).

4 Cheng, Q. F., Li, M. Z., Yang, F., Liu, M. J., Li, L., Wang, S. T. \& Jiang, L. An underwater pH-responsive superoleophobic surface with reversibly switchable oil-adhesion. Soft Matter 8, 6740-6743 (2012). 
5 Duong, H. T. T., Marquis, C. P., Whittaker, M., Davis, T. P. \& Boyer, C. Acid degradable and biocompatible polymeric nanoparticles for the potential codelivery of therapeutic agents. Macromolecules 44, 8008-8019 (2011).

6 Hoenders, D., Tigges, T. \& Walther, A. Combining the incompatible: block copolymers consecutively displaying activated esters and amines and their use as proteinrepellent surface modifiers with multivalent biorecognition. Polymer Chem. 6, 476-486 (2015).

7 Arnold, R. M. \& Locklin, J. Self-sorting click reactions that generate spatially controlled chemical functionality on surfaces. Langmuir 29, 5920-5926 (2013).

8 Arnold, R. M., McNitt, C. D., Popik, V. V. \& Locklin, J. Direct grafting of poly(pentafluorophenyl acrylate) onto oxides: versatile substrates for reactive microcapillary printing and self-sorting modification. Chem. Commun. 50, 5307-5309 (2014).

9 Zhao, H. Gu, W. Thielke, M. W. Sterner, E. Tsai, T, Russell, T. P. Coughlin, E. B. \& Theato, P. Functionalized nanoporous thin films and fibers from photocleavable block copolymers featuring activated esters. Macromolecules 46 , 5195-5201 (2013).

10 Seo, J., Schattling, P., Lang, T., Jochum, F., Nilles, K., Theato, P. \& Char, K. Covalently bonded layer-by-layer assembly of multifunctional thin films based on activated esters. Langmuir 26, 1830-1836 (2010).

11 Jang, Y., Akgun, B., Kim, H., Satija, S. \& Char, K. Controlled release from model blend multilayer films containing mixtures of strong and weak polyelectrolytes. Macromole cules 45, 3542-3549 (2012).
12 Jang, Y., Seo, J., Akgun, B., Satija, S. \& Char, K. Molecular weight dependence on the disintegration of spin-assisted weak polyelectrolyte multilayer films. Macromolecules 46, 4580-4588 (2013).

13 Papanu, J. S., Soane, D. S., Bell, A. T. \& Hess, D. W. Transport models for swelling and dissolution of thin polymer films. J. Appl. Polym. Sci. 38, 859-885 (1989).

14 Pekcan, Ö., Canpolat, M. \& Kaya, D. In situ fluorescence experiments for real-time monitoring of annealed high-T latex film dissolution. J. Appl. Polym. Sci. 60 2105-2112 (1996).

15 Miller-Chou, B. A. \& Koenig, J. L. A review of polymer dissolution. Prog. Polym. Sci. 28, 1223-1270 (2003)

16 Sivaniah, E., Hayashi, Y., lino, M., Hashimoto, T. \& Fukunaga, K. Macromolecules 36, 5894 (2003).

17 Eberhardt, M., Mruk, R., Zentel, R. \& Théato, P. Synthesis of pentafluorophenyl(meth) acrylate polymers: new precursor polymers for the synthesis of multifunctional materials. Eur. Polym. J. 41, 1569-1575 (2005).

18 Choi, J., Schattling, P., Jochum, F. D., Pyun, J., Char, K. \& Theato, P. Functionalization and patterning of reactive polymer brushes based on surface reversible addition and fragmentation chain transfer polymerization. J. Polym. Sci. Part A Polym. Chem. 50, 4010-4018 (2012).

19 Sauerbrey, G. Verwendung von schwingquarzen zur wagung dunner schichten und zur mikrowagung. Z. Phys. 155, 206-222 (1959).

20 Fetters, L. J., Lohse, D. J., Colby, R. H. in Physical Properties of Polymers Handbook (ed. Mark, J.) Ch. 25, 447-454 (Springer, New York, NY, USA, 2007). 